\title{
Begleitung anspruchsvoller Bildungswege: Coaching für Studierende
}

\section{Matthias Wiemer}

Zusammenfassung: An Hochschulen existieren vielfältige Beratungsangebote für Studierende, die zunehmend auf das Format Coaching zurückgreifen. Der Beitrag zeigt auf, welche Zielgruppen mit Coaching erreicht und welche Anlässe im Beratungsformat Coaching bearbeitet werden können. Ein besonderes Augenmerk liegt darüber hinaus auf studierendenzentrierter Lehre und Kompetenzentwicklung an Hochschulen und wie diese Aspekte durch Coaching (elemente) unterstützt werden können.

Schlüsselwörter: Coaching $\cdot$ Studierende $\cdot$ Kompetenzentwicklung

\section{Guidance for challenging educational pathways: coaching for students}

\begin{abstract}
The institutions of higher education offer various advisory and consulting services for students, which increasingly rely on the counseling format coaching. The article shows which students can be reached and with which kind of needs and occasions can be dealt with in the scope of coaching. Furthermore, a special focus lies on student-oriented teaching and competency development in higher education and how these issues can be supported through coaching.
\end{abstract}

Keywords: Coaching $\cdot$ Students $\cdot$ Competency development in higher education

Online publiziert: 21.02 .2012

(C) Die Autor(en) 2012. Dieser Artikel ist auf Springerlink.com mit Open Access verfügbar.

M. Wiemer $(\bowtie)$

Hochschuldidaktik, Georg-August-Universität Göttingen,

Waldweg 26, 37073 Göttingen, Deutschland

E-Mail: matthias.wiemer@zvw.uni-goettingen.de 


\section{Einleitung: Beratungsangebote für Studierende}

Beratungsangebote für Studierende sind an Hochschulen fest etabliert und nach Anliegen und Zielgruppen ausdifferenziert: Zentrale Studienberatungsstellen, Studienfachberatung, psychologisch-soziale Studienberatung, Fachschaften, Career Services und weitere institutionell verankerte Angebote stehen den Anliegen der Studierenden zur Verfügung. Gemeinsam ist den Angeboten, dass sie sich an eben diese Zielgruppe richten: Studierende (oder zumindest zukünftige Studierende). Die jeweilige Ausgestaltung der Angebote reagiert dabei auf konkrete kollektive und individuelle Anfragen und Bedarfe der Studierenden. Sie reicht von der bloßen Bereitstellung von Informationen über punktuelle Einzelberatungen und Entscheidungshilfen (zur Studien- und Berufswahl etc.) bis hin zu verschiedenen Möglichkeiten des Trainings, etwa in Bezug auf fächerübergreifende Schlüsselqualifikationen. Darüber hinaus stehen Angebote zur Unterstützung bei der Bewältigung von Krisen zur Verfügung, die im Zusammenhang mit dem Studium stehen bzw. das Studium beeinflussen können, z. B. bei Prüfungsangst (zum Praxisfeld Beratung an Hochschulen vgl. Großmaß und Püschel 2006).

Im Zuge einer in vielen Beratungsbereichen konstatierbaren Verbreitung des Coachingbegriffs werden seit einigen Jahren auch zunehmend Angebote für Studierende mit dem Label Coaching belegt. In diesem Beitrag möchte ich aufzeigen, was Coaching für Studierende bedeuten kann. Zentrale Fragstellungen sind,

- in welchen Kontexten und für welche Zielgruppen ein solches Beratungsformat angeboten wird,

- wie es um die Zusammenhänge von Coaching und Lehre steht und

- was Coaching für Studierende leisten kann und soll.

\section{Zielgruppen und Anlässe}

Coaching ist ein personen- und prozessorientiertes Beratungsformat, das - ausgehend von den Ressourcen, Zielen und der konkreten Situation des Coachees (des Ratsuchenden) auf die Förderung der Leistungsfähigkeit bei der Bewältigung anspruchsvoller Aufgaben abzielt. Zentraler Aspekt ist hier die Aktivierung eines größeren Spielraums an Möglichkeiten und die Entwicklung eigener, situations- und personenadäquater Lösungen und Lösungsansätze, die der Coach durch verschiedene Verfahren und Methoden unterstützt. Damit verbunden ist dementsprechend die Selbst- und Rollenreflexion im Hinblick auf die Durchführung spezifischer Aufgaben im konkreten, vor allem beruflichen, aber auch privaten Kontext (zum Begriff des Coachings vgl. exemplarisch Rauen 2005).

Die Attraktivität zielorientierter Arbeit im Spannungsfeld von Selbst, Rolle und Institution/Kontext erklärt, warum der Begriff des Coachings sich seit einer Reihe von Jahren großer Beliebtheit erfreut und für eine Vielzahl verschiedener Beratungsangebote in Anspruch genommen wird. Neben den Risiken und Fragen, die mit der teilweise fast inflationären Verwendung dieses Begriffs zusammenhängen und in den notwendigen Diskussionen um Standards und Definitionen resultieren, zeigen sich in der weiten Verbreitung ebenfalls bestimmte Bedürfnisse der Ratsuchenden, wie der begründete Wunsch 
nach individueller und persönlicher Beratung und einer gewissen Zielgruppenorientierung, aber auch nach Leistungsorientierung und Zielklarheit, ebenso wie nach Wertschätzung und einer gewissen Exklusivität. Der Begriff Coaching kann diese Bedürfnisse offensichtlich besser abdecken, als es für andere Beratungsformate der Fall ist: etwa durch die Nähe zum Spitzensport in Bezug auf Leistungsfähigkeit, oder in Bezug auf Exklusivität, insofern Coaching insbesondere als Beratungsformat für Führungskräfte oder allgemeiner für Personen in Leitungsfunktion wahrgenommen wird. Zentral scheint hier auch zu sein, dass der Begriff eine auf die individuelle Person und Persönlichkeit zurechtgeschnittene Beratungskommunikation verspricht, der der Ruch nach psychosozialer Beratung jedoch nicht anhaftet (Schuhmann 2008, S. 51). Coaching verspricht Orientierung in einer schnelllebigen Gesellschaft und in sich wandelnden Institutionen und Arbeitsfeldern durch die Möglichkeit einer zielorientierten Vorbereitung und Förderung zu erbringender individueller Leistungen. Dies gelingt durch die systematische Verschränkung von ,individueller Beratung, persönlichem Feedback und praxisorientiertem Training“ (Fischer-Epe 2011, S. 19).

Unter diesen Gesichtspunkten wird deutlich, dass Coaching als Instrument „,zur Verbesserung der persönlichen Effektivität“" (Greif 2005, S. 7) viele Chancen und einen großen Gestaltungsspielraum bereitstellt; als eine personenbezogene Beratung, die auf Unsicherheitsreduzierung, Steigerung der Leistungsfähigkeit, Vorbereitung auf neue Aufgaben und Bewältigung von Sinnkrisen abzielt, kann Coaching für viele Zielgruppen ein reizvolles Angebot darstellen. Eine Fokussierung nur auf einzelne Zielgruppen, etwa Führungskräfte, verstellt hingegen viele Möglichkeiten.

Auch Studierende unterliegen den Zwängen und Herausforderungen gesellschaftlicher und institutioneller Erfordernisse, denen sie nicht immer adäquat begegnen können. Der Umgang z. B. mit Leistungsdruck und der im Studium geforderten Flexibilität innerhalb häufig starrer Rahmenbedingungen gehört nicht in dem Maße (wenn überhaupt) zum Lehrplan der Schulen, dass alle Studieneinsteiger/innen ohne zusätzliche Unterstützungsangebote problemlos an der Hochschule Fuß fassen können. Mit dem Übergang Schule-Studium sind neue Leistungsanforderungen, vielfältige neue Aufgaben wissenschaftlichen Arbeitens verknüpft, die allein schon eine Herausforderung darstellen. Darüber hinaus aber gestaltet sich dieser Übergang zumeist als ein großer persönlicher Wendepunkt, wenn der Auszug aus dem elterlichen Zuhause, ein Wohnortswechsel und die Suche nach neuen sozialen Kontakten damit verknüpft sind. Und auch während des gesamten Studiums bleiben die Herausforderungen durch neue Aufgaben und vielfältige Übergänge bestehen, potenzieren sich in Prüfungssituationen und in der Frage nach der beruflichen Karriere, die mit dem Studienabschluss beginnt.

Konkretisierend wären hier beispielsweise weitere Übergangssituationen zu nennen, die das Studium mit sich bringt (Bachelor-Master, Studium-Beruf, aber auch Beruf-Studium), die zielorientierte Planung des Studiums, Zeit- und Selbstmanagement (Ebner 2009), die Studienabschlussphase (Schuhmann 2008) oder Schreibaufgaben und Prüfungssituationen (Wildt und Redecker 2008). Mit diesen Anlässen findet die Etablierung von entsprechenden Coachingangeboten für Studierende seine Berechtigung. Darüber hinaus lassen sich verschiedene Zielgruppen in der Studierendenschaft für ein Coaching identifizieren, für die an verschiedenen Hochschulen auch bereits ein Coaching-Angebot besteht: 
- Studierende mit besonderen familiären Situationen (z. B. Studierende mit Kind, Pflege von Angehörigen), die Unterstützung und Beratung für die damit verbundenen besonderen Belastungen suchen;

- Tutor/innen, die durch Coaching bei der Einfindung in die neue Rolle als Studierende mit Lehrfunktion und bei der (durchaus konfliktträchtigen) Arbeit mit den Kommilitonen und Lehrenden begleitet werden (vgl. Knauf 2006);

- Lehramtsstudierende, die auf den anstehenden Rollenwechsel vorbereitet werden und im Gruppencoaching ,soziale[s] Lernen durch Perspektivwechsel“ erfahren (Reis 2006, S. 167);

- Internationale Studierende, die bei der Einfindung in vielfältige neue kulturelle Zusammenhänge unterstützt werden.

Gelingensbedingung für das Coaching von Studierenden ist dabei stets das Prinzip der Freiwilligkeit, insofern die im Coaching vorgenommene Arbeit an Einstellungen und Haltungen einen Veränderungswillen bei den Studierenden voraussetzt, dem aufgezwungene Beratung (selbst wenn sie gutmeinend als Zwangsbeglückung verstanden wird) zuwiderläuft. Coachingangebote für Studierende können damit nur als eine Ergänzung des Beratungsangebots an Hochschulen verstanden werden und nicht als Ersatz. Gerade an Massenuniversitäten, die einen hohen Anonymitätsgrad aufweisen, lassen sich durch Coaching zumindest im Kleinen geschützte Räume etablieren, die eine persönliche und vertraute Beratungsatmosphäre ermöglichen. Wenn es sich in vielen Fällen aufgrund der Rahmenbedingungen dabei um Gruppencoachings (vgl. Rückle 2005) handelt, ist dies in Bezug auf die Anonymität und das häufig feststellbare Einzelkämpfertum des Studiums durchaus wünschenswert.

Ebenfalls stark beeinflusst durch die gegebenen Rahmenbedingungen an Hochschulen und die Situation der Studierenden ist die Auswahl der Coaches: Hier können Studierende zwischen Angeboten externer Coaches, die die Zielgruppe Studierende im Auge haben, und hochschulinternen Angeboten auswählen, wobei die Inanspruchnahme eines externen Coachs aufgrund der finanziellen Belastung für die Studierenden wohl die Ausnahme ist. Hochschulintern sind neben den professionellen Beratungsstellen mittlerweile auch einige Peer- (z. B. Ebner 2009) und Online-Coaching-Angebote (z. B. Minckert und Böhning 2009) zu finden.

\section{Coaching in der Lehre}

Verstärkt wird der Coachingbegriff auch im Kontext der Lehre an Hochschulen verwendet, z. B. als in die Lehre integrierte Lernberatung. Ausgehend von einem durch die Bologna-Reformen geforderten Wandel der Lehr-Kultur, von der Instruktion zur Begleitung studentischen Lernens, erweitern sich die Handlungsebenen professioneller Lehre an Hochschulen: Neben der Präsentation des Fachwissens und dem Arrangement der Lernumgebung für den angemessenen Umgang mit diesem Wissen soll professionelle Lehre die Studierenden motivieren, die Selbstreflexion der Studierenden anregen und helfen, Lernblockaden zu vermeiden und wenn möglich zu überwinden (Szczyrba 2006).

Diese Ebenen vorausgesetzt, kann der Kurzschluss Lehre=Coaching recht schnell folgen. Die Rede von Lerncoaching (vgl. Schumacher 2007), vom Dozenten als Coach 
(Blom 2000) ist auch in weiterem Sinne durchaus berechtigt. Denn insofern Coaching immer auch die Begleitung eines Coachees bei der Leistungsvorbereitung und -förderung bedeutet, ist es idealerweise genau dies, was von Lehrenden an Hochschulen verlangt wird. Studierende sollen durch die Gestaltung geeigneter Lernumgebungen, den Einsatz interaktionsfördernder und aktivierender Methoden „beim Erwerb studiengangsspezifisch definierter Kompetenzen (fachwissenschaftliche, fachübergreifende, methodische, strategische etc.) optimal“" (HRK 2010, S. 2) durch die Lehrenden unterstützt und darauf vorbereitet werden, in eine berufliche oder wissenschaftliche Laufbahn einzutreten. Um dieses Ziel zu erreichen, sollen die Lehrenden den Dialog mit den Studierenden als „selbständige, eigenverantwortliche Lerner“ suchen, ihnen „Orientierungshilfen“ und „,individuelle Entfaltungsmöglichkeiten“ bieten (HRK 2008, S. 3 f.); dazu ist laut Hochschulrektorenkonferenz unter anderem „systematisches und regelmäßiges“ Feedback (ebd. S. 4) der Schlüssel. Bezogen auf die Lehrtätigkeit und die Vorbereitung auf die Erbringung spezifischer Leistungen (Methodik, wissenschaftliche Arbeitssystematik, Prüfungen etc.) sind die Aufgaben von Lehrenden also durchaus mit denen eines Coachs zu vergleichen, vor allem, wenn das Studium unter dem Aspekt der Kompetenzentwicklung betrachtet wird.

Im Kompetenzbegriff sind immer auch personenbezogene Aspekte mit einbezogen, insofern es nicht nur um ein Können, sondern um individuelles, verantwortungsvolles und angemessenes Handeln in komplexen Situationen geht (vgl. Reis 2009). Gerade der Aspekt der Person scheint in vielen Lehrveranstaltungsangeboten an Hochschulen keine Rolle zu spielen, sind die Studierenden doch für diesen Aspekt ihrer persönlichen und auch wissenschaftlichen Entwicklung offenbar ganz selbst zuständig und der Verantwortungsbereich der Hochschullehrer/innen und der Dozent/innen endet hier vermeintlich. Ziehen wir die oben genannten Forderungen der Hochschulrektorenkonferenz jedoch hinzu, zeichnet sich ein anderes Bild. Lehrende und andere Personen mit Beratungsauftrag an Hochschulen sind selbstverständlich nicht in der Verantwortungsposition von Eltern oder Erziehern, aber durchaus in der Verantwortungsposition von (Lern-)Prozessbegleitern. Dazu gehört dann auch, den Selbstverantwortungsraum der Studierenden durch Anregung von selbstreflexiven Prozessen ggf. erst einmal bewusst zu machen und diesen Entwicklungsprozess innerhalb der Beziehung Wissenschaft - Person konstruktiv zu begleiten. Der häufig anzutreffende Fokus auf den Pol der Wissenschaft, auf die fachlichen Anforderungen und Inhalte, schließt einen für die Studierenden wichtigen Aspekt ihres Studiums aus, das sie selbst als ,eine wichtige Phase der persönlichen Entwicklung und Selbstfindung als Experte/Expertin in dem gewählten Fach“ betrachten (Berthold et al. 2011, S. 3). Damit wird das auf den Kompetenzerwerb ausgerichtete Studium als Teil eines offenen Prozesses verstanden, der nicht mit dem Hochschulabschluss beendet ist.

Mit dem Beginn dieses Prozesses ist der Eintritt in die Wissenschaftskultur und der jeweiligen Fachkultur(en) und damit das Einfinden in neue Rollen verbunden, in denen Studierende nicht als Wissenskonsumenten, sondern als aktive und verantwortungsbewusste (Ko-)Produzenten von Wissen agieren. Hier ist dann tatsächlich der Lehrende als Coach gefragt, der neben der Instruktion und der Präsentation fachlichen Wissens den Studierenden praxisorientiertes Training ermöglicht, persönliches Feedback gibt und individuelle Beratung angedeihen lässt; Aufgaben, die dem oben genannten Coachingverständnis von Fischer-Epe entsprechen. 
Andererseits - und hier hakt das Bild vom Dozenten als Coach - lässt sich die Hierarchie zwischen Lehrenden und Studierenden nicht gänzlich zugunsten einer Begegnung auf Augenhöhe aufheben, und die Lehre an Hochschulen beinhaltet mehr als eine auf Kompetenzorientierung und Rolleneinfindung ausgerichtete Prozessbegleitung. Einige der Aufgaben von Lehrenden widersprechen dem Verständnis von Coaching dabei direkt, da sie z. B. die Bewertung von Prüfungsleistungen beinhalten. Auch ist fraglich, ob auf das Prinzip der Freiwilligkeit in Pflichtveranstaltungen rekurriert werden kann. Darüber hinaus sind Lehrende natürlich keine ausgebildeten Coaches; wenn aber der Vergleich Lehre - Coaching neue Erkenntnisse fördert, Einstellungen und Haltungen offenbar macht oder auch nur zur Selbstreflexion der Lehrenden in ihrer Rolle als Lernbegleiter und -berater anregt, sollte man ihn wegen des häufigen (und oft unreflektierten) Einsatzes des Coachingbegriffs nicht unterlassen. Hier wäre allerdings eine systematische hochschuldidaktische und auf Beratungskommunikation und -formate ausgerichtete Weiterbildung der Lehrenden wünschenswert, die deren Lehrkompetenzentwicklung gewährleisten und zur Durchsetzung einer studierendenzentrierten und lernorientierten Lehre an Hochschulen beitragen könnte.

\section{Coaching für Studierende}

Coaching, ob für Studierende oder andere Zielgruppen, ist (und so soll es auch bleiben) ein professionelles Beratungsformat, das von ausgebildeten Berater/innen durchgeführt werden sollte. Das bedeutet aber nicht, dass die Möglichkeiten des Coachings, Lehre studierendenorientierter auszurichten und Studierende auf ihren anspruchsvollen Bildungswegen zu begleiten, verstellt sein sollten und dass auf Coaching als Impuls- und Ideengeber, als Perspektive und Unterstützung für Lehre und Beratung an Hochschulen zu verzichten wäre.

Kompetenzentwicklung braucht auf Einstellungen und Haltungen fokussierte Selbstreflexionsprozesse, um nachhaltige und langfristige Lernprozesse auf Seiten der Studierenden anzuregen und die Unsicherheiten, die im Prozess des Kompetenzerwerbs entstehen können, zu reduzieren. Dies meint mehr, als die Studierenden auf die fachlichen Anforderungen vorzubereiten, und bedeutet eben nicht, die Studierenden bei der Hand zu nehmen und ihnen den einen, (vermeintlich) richtigen Weg zu zeigen. Es heißt, ihnen Optionen und Möglichkeiten bereitzustellen, einen eigenen Weg durchs Studium und im Feld der Wissenschaft zu finden, ihnen Orientierungsmöglichkeiten aufzeigen und mit ihnen Mittel und Wege zu erarbeiten, die Herausforderungen des Studienalltags erfolgreich zu bewältigen, um Vertrauen in die eigene Kompetenz zu erlangen. ${ }^{1}$

Die Notwendigkeit dafür lässt sich aus aktuellen Ergebnissen einer Befragung von Studierenden und Professor/innen bezüglich der Perspektiven auf das Studium (Berthold et al. 2011) ableiten, die zeigen, dass die Bewertungen der Herausforderungen im Studium

1 Wenn dagegen die verschiedenen Herausforderungen, die das Studium mit sich bringt, auf Seiten einzelner Studierender zu tiefgreifenden persönlichen Belastungen der psychischen Gesundheit und Blockaden geführt hat, kann es nicht Aufgabe der Studienberater/innen und der Lehrenden sein, diese zu lösen und zu bearbeiten. 
zwar jeweils Diskrepanzen aufweisen, beide Gruppen aber Zeitmanagement, den Erhalt einer hohen Motivation (keine „Konsumhaltung“), die Konzentration auf das Studium (trotz Nebenjobs, sozialen oder familiären Verpflichtungen, Freizeitaktivitäten, ...) und die Aneignung einer wissenschaftlichen Arbeitshaltung als wichtigste Herausforderungen im Studium bewerten (ebd. S. 22 ff.). Studieren unter Bologna-Bedingungen und mit den gesellschaftlichen Anforderungen der Gegenwart ist eine anspruchsvolle Aufgabe, die eine hohe Beratungsleistung auf Seiten der Hochschulen erfordert. Hier kann Coaching für Studierende wertvolle Dienste leisten, da es die Möglichkeiten für notwendige Perspektivenwechsel, für das Einfinden in neue Rollen und Zusammenhänge und das Einüben einer wissenschaftlichen Haltung schon in der Lehre bereitstellt und zur Steigerung der Leistungsfähigkeit und Optimierung der Work-Life-Study-Balance in lehrveranstaltungsexternen Coachingangeboten beitragen kann. Dass dies eine Steigerung der Studierfähigkeit impliziert und damit eine Steigerung der Zufriedenheit und Motivation sowohl von Studierenden als auch von Lehrenden einhergeht, steht zu vermuten.

Die aufgezeigten Möglichkeiten und Anlässe abschließend zusammenführend, lässt sich Coaching für die Zielgruppe Studierende als ein auf die Arbeit an Einstellungen und Haltungen bezogenes, personen- und (lern)prozessorientiertes Beratungsformat charakterisieren, dem sich mit dem Studium und der Kompetenzentwicklung der Studierenden ein breites Feld eröffnet (vgl. Abb. 1):

Mit Rott lässt sich das hier umrissene Spannungsfeld auf die Aspekte der inneren und äußeren Welt und die Balance zwischen individueller Persönlichkeitsentwicklung und Anpassungsprozessen an die sozialen, gesellschaftlichen und beruflichen Gegebenheiten zurückführen, die bei der Gestaltung von Beratungsangeboten bedeutsam sind: „Erstens sehen sowohl der Begriff ,Kompetenz' als auch die Nachfrage nach fachübergreifenden, übertragbaren Fähigkeiten den einzelnen Studierenden als eine Person, die Wissen in Handlung umsetzen kann. Unser Augenmerk wird verstärkt darauf gelenkt, dass die Integration von komplexen umwelt- und intellektuell bedingten Anforderungen in Richtung auf Handlungskompetenz starken Einfluss auf die Persönlichkeitsentwicklung hat. Zweitens verlangt das Konzept der Beschäftigungsfähigkeit von den Studierenden, sich in die Zukunft zu projizieren. Es wird zu ihrer Aufgabe, gegenwärtige Anpassungsprozesse und Persönlichkeitsentwicklung als eine Voraussetzung für zukünftige Adaption zu begreifen“ (Rott 2007, S. 35).

Coachingangebote bieten die Möglichkeit, Studierende bei der Gestaltung dieses offenen Prozesses der Kompetenz- und Persönlichkeitsentwicklung zu begleiten und das Studium zu einer nachhaltigen und erfolgreichen (Lern-)Erfahrung zu machen.

Abb. 1: Coaching als Arbeit an Einstellungen und Haltungen im Spannungsfeld Studium

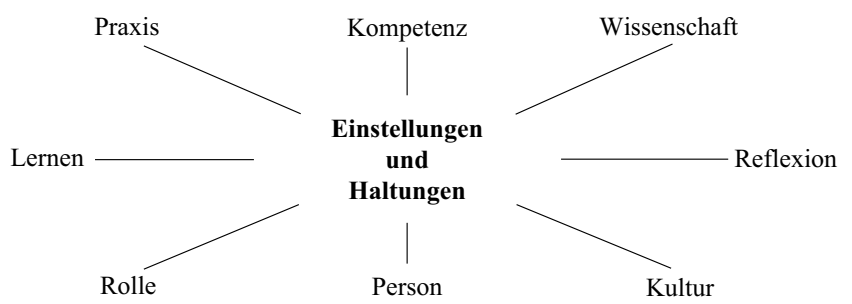


Open Access: Dieser Artikel unterliegt den Bedingungen der Creative Commons Attribution License. Dadurch sind die Nutzung, Verteilung und Reproduktion erlaubt, sofern der/die Originalautor/en und die Quelle angegeben sind.

\section{Literatur}

Berthold, C., Kessler, M. S., Kreft, A.-K., \& Leichsenring, H. (2011). Schwarzer Peter mit zwei Unbekannten. Ein empirischer Vergleich der unterschiedlichen Perspektiven von Studierenden und Lehrenden auf das Studium (CHE Arbeitspapier Nr. 141). http://www.che.de/downloads/ CHE_AP141_Doppelbefragung.pdf. Zugegriffen: 5. Okt. 2011.

Blom, H. (2000). Der Dozent als Coach. Neuwied: Luchterhand.

Ebner, K. (2009). Entwicklung der Studierfähigkeit als Aufgabe der Universität. Coaching studentischer Selbstmanagementkompetenzen. Zeitschrift für Hochschulentwicklung, 4(3), 37-52.

Fischer-Epe, M. (2011). Coaching: Miteinander Ziele erreichen (vollst. überarb. Neuausg.). Reinbek: Rowohlt.

Greif, S. (2005). Mehrebenencoaching von Individuen, Gruppen und Organisationen. Beitrag zum CoachingKongress 2005, Frankfurt a. M. http://www.home.uni-osnabrueck.de/sgreif/downloads/Mehrebenencoaching.pdf. Zugegriffen: 5. Okt. 2011.

Großmaß, R., \& Püschel, E. (2006). Hochschulberatung als eigenständiges Praxisfeld. Feldspezifik, Merkmale, Besonderheiten. Zeitschrift für Beratung und Studium, 1(4), 113-118.

Hochschulrektorenkonferenz (HRK). (2008). Für eine Reform der Lehre in den Hochschulen (3. Mitgliederversammlung der HRK am 22 April 2008). http://www.hrk.de/de/download/dateien/ Reform_in_der_Lehre_-_Beschluss_22-4-08.pdf. Zugegriffen: 5. Okt. 2011

Hochschulrektorenkonferenz (HRK). (2010). Weiterführung der Bologna-Reform. Kontinuierliche Qualitätsverbesserung in Lehre und Studium (Entschließung der 8. Mitgliederversammlung der HRK am 11 Mai 2010). http://www.hrk.de/de/download/dateien/MV_8-_3_Entschliessung_Weiterfuehrung_Bologna-Reform.pdf. Zugegriffen: 5. Okt. 2011.

Knauf, H. (2006). Vom Frosch zum Adler. Wie Tutorinnen und Tutoren ihre Arbeit durch Coaching verbessern können. In J. Wildt, B. Szczyrba, \& B. Wildt (Hrsg.), Consulting, Coaching, Supervision. Eine Einführung in Formate und Verfahren hochschuldidaktischer Beratung (S. 203214). Bielefeld: WBV.

Minckert, U., \& Böhnert, M. (2009). Das Online-Coaching-Portal ,UPrepare‘ im Akademischen Auslandsamt der Universität Potsdam. Eine Zwischenbilanz. Zeitschrift für Beratung und Studium, 4(2), 54-59.

Rauen, C. (Hrsg.). (2005). Handbuch Coaching (3., überarb. u. erw. Aufl). Göttingen: Hogrefe.

Reis, O. (2006). Gruppencoaching für Lehramtsstudierende. Ein leistungsfähiger Beitrag zur Studiengangsreform. In J. Wildt, B. Szczyrba, \& B. Wildt (Hrsg.), Consulting, Coaching, Supervision. Eine Einführung in Formate und Verfahren hochschuldidaktischer Beratung (S. 158-173). Bielefeld: WBV.

Reis, O. (2009). Durch Reflexion zur Kompetenz. Eine Studie zum Verhältnis von Kompetenzentwicklung und reflexivem Lernen an der Hochschule. In R. Schneider, B. Szczyrba, U. Welbers, \& J. Wildt (Hrsg.), Wandel der Lehr- und Lernkulturen (S. 100-120). Bielefeld: WBV.

Rott, G. (2007). Wissenserwerb und Kompetenzentwicklung im Studium - neue Rollenanforderungen. Zeitschrift für Beratung und Studium, 2(2), 31-36.

Rückle, H. (2005). Gruppen-Coaching. In C. Rauen (Hrsg.), Handbuch Coaching (3., überarb. u. erw. Aufl., S. 183-198). Göttingen: Hogrefe.

Schuhmann, B. (2008). SCHLUSS.PUNKT. Studienabschlusscoaching. Damit das Studium nicht zur unendlichen Geschichte wird. Zeitschrift für Beratung und Studium, 3(2), 51-54. 
Schumacher, E.-M. (2007). Lerncoaching. Lernumgebungen gestalten - Studierende coachen. In B. Berendt, H.-P. Voss, \& J. Wildt (Hrsg.), Neues Handbuch Hochschullehre (Griffmarke A 3.5). Berlin: Raabe.

Szczyrba, B. (2006). Instruieren, Arrangieren, Motivieren ... Handlungsebenen professioneller Lehre. In B. Berendt, H.-P. Voss, \& J. Wildt (Hrsg.), Neues Handbuch Hochschullehre (Griffmarke A 3.3). Berlin: Raabe.

Wildt, B., \& Redecker, D. (2008). „Prüfungscoaching“ - zur Vorbereitung von Studierenden auf die mündliche Prüfung: ein Werkstatt-Konzept. In S. Dany, B. Szczyrba, \& J. Wildt (Hrsg.), Prüfungen auf die Agenda! Hochschuldidaktische Perspektiven auf Reformen im Prüfungswesen (S. 200-217). Bielefeld: WBV.

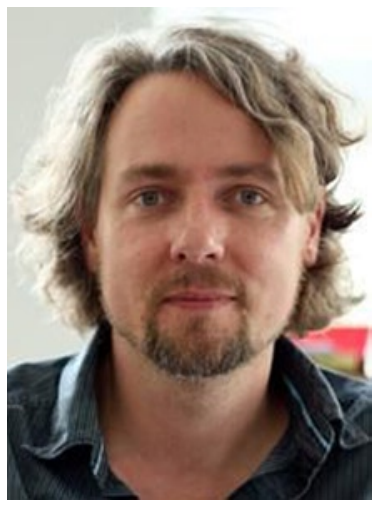

Matthias Wiemer, Kulturwissenschaftler und hochschuldidaktischer Multiplikator, leitet die Hochschuldidaktik an der GeorgAugust-Universität Göttingen. Er war Sprecher des Netzwerks Tutorienarbeit an Hochschulen und ist Mitglied im Netzwerk Wissenschaftscoaching. 